\title{
Optimization of the Multimedia Consumers Operation Time
}

\author{
Mihai Oproescu \\ Faculty of Electronics, \\ Communications and \\ Computers \\ University of Pitesti \\ Pitesti, Romania \\ mihai.oproescu@upit.ro \\ Cristian Monea \\ Doctoral School of Electronics, \\ Telecommunications and \\ Information Technology \\ University Politehnica of \\ Bucharest \\ Bucharest, Romania \\ cristianmonea0@gmail.com
}

\author{
Gabriel V. Iana \\ Faculty of Electronics, \\ Communications and \\ Computers \\ University of Pitesti \\ Pitesti, Romania \\ gabriel.iana@upit.ro \\ Valentin Calinescu \\ Faculty of Electronics, \\ Communications and \\ Computers \\ University of Pitesti \\ Pitesti, Romania \\ vali.calinescu97@yahoo.com
}

\author{
Nicu Bizon \\ Faculty of Electronics, \\ Communications and \\ Computers \\ University of Pitesti \\ Pitesti, Romania \\ nicu.bizon@upit.ro
}

\begin{abstract}
This article analyses the consumption of household multimedia equipment. This comes to the aid of parents who want to limit the time their children spend in front of the TV, computer or using game consoles. The novelty of the presented solution consists in limiting the access to the energy sources by identified the multimedia equipment coupled to the power supply and not just to monitor the consumption. Also, this solution allows a timetable programmed by the user that is dependent on the connected time of the multimedia equipment, as it will be explained in the paper. In order to benefit from all its functions, the proposed device must be embedded in the wall socket. Thus it cannot be removed, and the monitored consumption is the correct one.
\end{abstract}

Keywords - multimedia equipment; power supply monitoring; electricity metering; conditioned access

\section{INTRODUCTION}

The first section of the paper presents the influence of multimedia equipment on the physical and mental health of the users. Recent studies show that long usage periods of smartphones do not cause cancer, but they seriously affect other body functions [1].

Starting with the posture-related problems and up to more serious problems such as impairing vision or metabolism-related, all these effects are worth considering, knowing that approximatively 6 billion people from a total of 7 billion plant inhabitants have used a smartphone in their lifetime and only 4.5 billion have access to a functional toilet.

\section{A. The computer: friend or enemy?}

Since its appearance, the computer has been controversial. Even though no one disputes the advantage of using a computer, the phenomenon of computer dependence appears more and more often these days. Used with caution, the computer can be our good friend [2,3]. Some of the advantages of using the computer by children can be:
- Learning to search and use information from different sources;

- Enriches their knowledge with information from various fields;

- Most games develop reaction speed, logical thinking and competitive spirit;

- By playing, they can learn the colors, numbers, letters, figures and geometric shapes;

- $\quad$ They learn to use an instrument that they will use in the years to come, maybe at their future job.

The irrational use of the computer also has disadvantages:

- Incorrect body position in front of the computer can lead to spine deformations;

- $\quad$ Lack of movement leads to obesity;

- Contact with unknown, dangerous persons;

- Viewing content that contains violent scenes or is forbidden for minors;

- $\quad$ Taking everything readymade;

- The tendency to use in conversations a language different from the real one;

- Creation of a distorted view of reality;

- It seems that some children become less social.

In order to correctly consider the solutions existing on the market, the second part of this paper presents some examples of electrical equipment used for monitoring and metering single-phase consumers. 


\section{SOLUTIONS FOR MONITORING AND/OR METERING EXISTING ELECTRICAL ENERGY}

Several electrical energy monitoring solutions have been developed over the course of time [adg]. Keeping up with the technological development, these monitoring solutions have started from mechanical variants to analog and now digital solutions $[4,5,6]$. Each product offers more or less the same facilities, but all help to measure the energy amount or to limit the usage time of the energy sources.

\section{A. Mechanical Programmable Outlet}

The first single-phase electrical energy consumption control solutions were represented by the mechanical programmable outlets. Such outlets, like TIMER-01-W (see Fig. 1), are powered at a voltage of $220-240 \mathrm{~V}$ and allow a maximum power of $3500 \mathrm{~W}$. The maximum current intensity is $16 \mathrm{~A}$ and the voltage's frequency is $50 \mathrm{~Hz}$.

Figure 1. TTIMER-01-W mechanical programmable outlet [7]

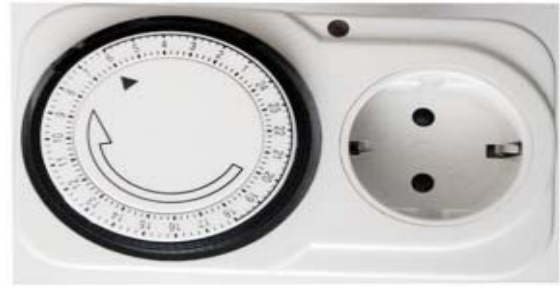

Characteristics:

- 24-hour operation;

- $\mathrm{ON} / \mathrm{OFF}$ program: 48 hours;

- $\quad$ Timing interval: $15 \mathrm{~min}$;

\section{B. Digital Programmable Outlet}

In line with the development of electronics and entering the digital era, digital monitoring and metering solutions have been developed, like the outlet presented in Fig. 2.

Figure 2. Digital programmable outlet [8]

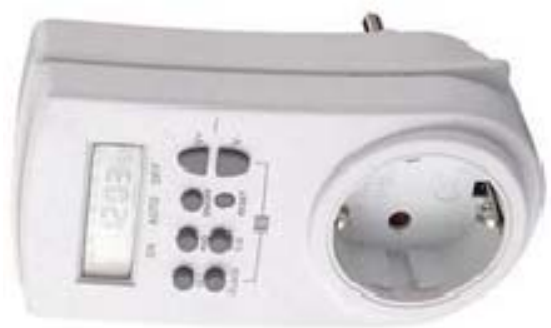

The digital programmable outlet is powered at a voltage of $230 \mathrm{~V}$, allow a maximum power of $3680 \mathrm{~W}$, with the operating frequency of $50 \mathrm{~Hz}$. The outlet is designed with an IP20 ingress protection rating. The main characteristics of this outlet are:

- Per-week programming at 1 min intervals;

- Maximum 98 start/stops per week;

- Countdown and random coupling.

\section{Smart Solution with Wireless Communication and Energy Consumption Monitoring and Metering}

The latest solutions on the market offer wireless communication with the device, besides the function to measure and/or monitor the electrical energy consumption.

Thus, remote control of equipment is allowed by the use of the Internet [9]. Also, multiple users can control the same outlet. With the help of the same smart device, one or more outlets can be controlled. The technical characteristics of such a device are:

- Overload and short-circuit protection;

- $\quad$ Maximum power: $3500 \mathrm{~W}$;

- The maximum power can be configured a value lower than $3500 \mathrm{~W}$ by the use of a software application, thus ensuring overload protection.

\section{HARDWARE AND SOFTWARE DESCRIPTION OF THE PROPOSED SOLUTION}

The solution that we are proposing integrates facilities from several equipment. Besides energy consumption monitoring and metering and temporary programming of a consumer's usage, the novelty we bring is given by the following: the user configures the usage times for each day of the week. Once the usage time for a given day is passed, the outlet can be used as soon as the next configured interval starts. Over the course of a day, the outlet monitors all consumers that are powered from it, but meters only those that pass a certain user-defined threshold.

E.g., if the outlet is configured to function for 2 hours on a Monday for a game console consumer, one the summed usage times for the console pass $120 \mathrm{~min}$, the console will be usable in the next day for which a usage interval is configured.

Figure 3. Hardware block diagram of the proposed system

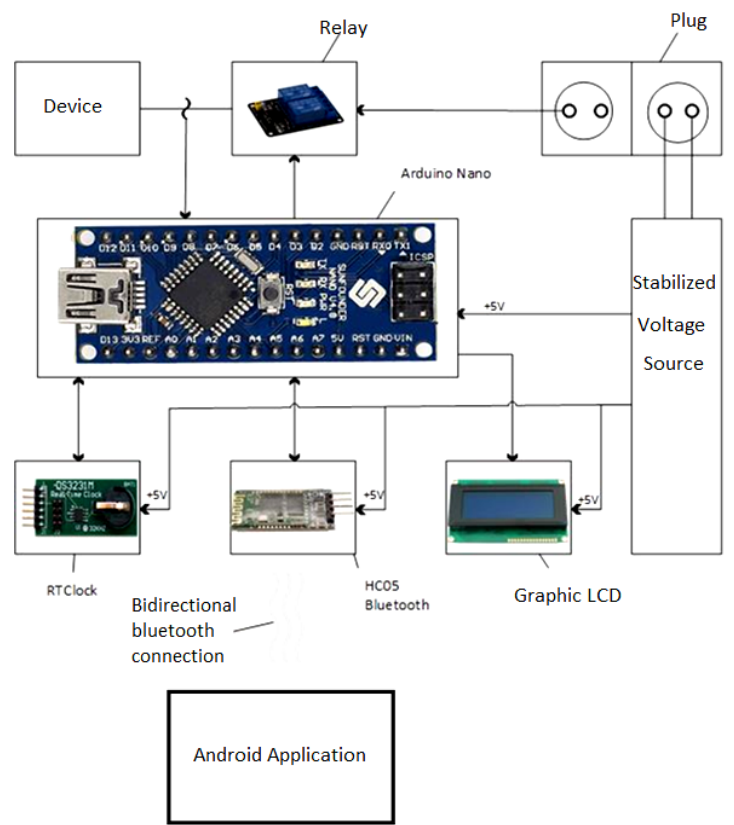


The block diagram illustrated in Fig. 3 contains:

- Regulated power supply: it provides the DC voltages required for the other blocks to operate;

- Arduino development board [10, 11]: it is powered from the $+5 \mathrm{~V}$ regulated power supply, it receives serial information via the Bluetooth module, it shows the information on a $128 \times 64$ px graphical LCD display and controls and monitors the RTClock block's real-time clock; also, it commands the relay module to connect or disconnect the power supply;

- Relay block: it receives the command to connect or disconnect the multimedia consumer;

- Consumer: it represents an electronic device (tablet, console, laptop, TV etc.) whose consumption is monitored and controlled;

- Bluetooth module: it receives information from the software application that is running on the smartphone or tablet and gives the values for the consumer's monitored parameters;

- RTClock: real-time clock that gives information regarding the date and time in order to ensure synchronization with the software application running in the Arduino;

- Android application: software application that sends the user-configured settings to the microcontroller via a bidirectional Bluetooth connection.

Since the hardware design consisted in the proper selection of modules and their integration in a functional system, the main focus is given to the software applications development.

There are two distinct software applications. One of them is installed on a smartphone, is developed in MIT AppInventor 2 and allows (as seen in the figures) the configuration of the time values for each day of the week, the creation of an array with these values, Bluetooth connection with the monitoring device and transmission of the array to a smart outlet $[12,13]$.

Figure 4. Android application interface

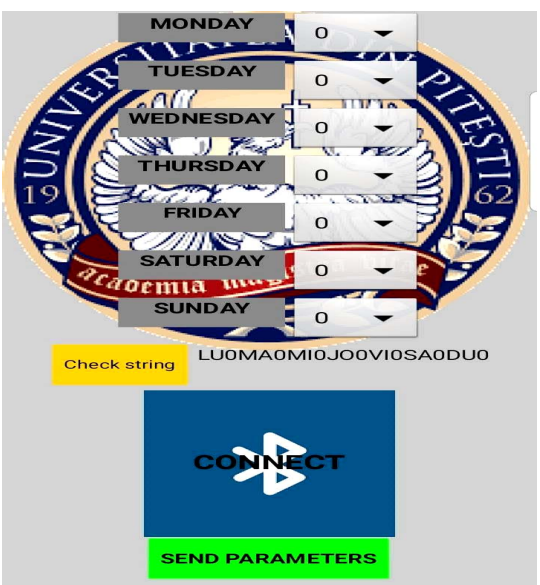

Figure 5. Transmission of the configured parameters to a smart outlet

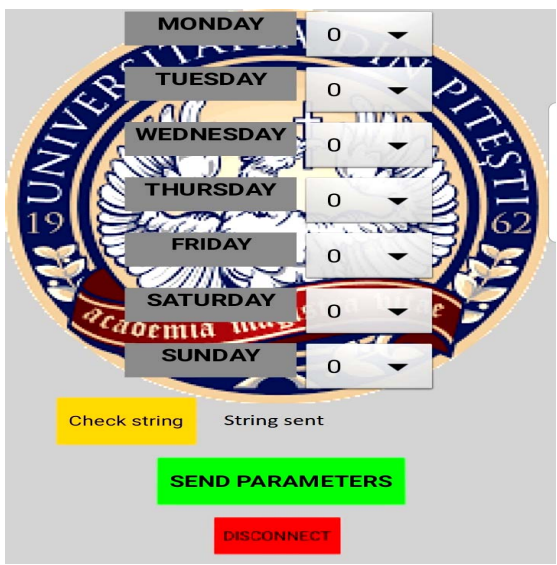

By pressing the "Connect" button (see Fig. 4), a previously created Bluetooth device list is called, thus allowing the selection of a device and establishing a connection to it. If the application successfully connects, the "Connect" vocal message is heard, that is defined using the function "call TextToSpeech.Speak message". Also, once the Bluetooth connection is established, the "Connect" button becomes invisible and the "Disconnect" button takes its place (see Fig. $5)$.

Figure 6. Code example for the Bluetooth connection function

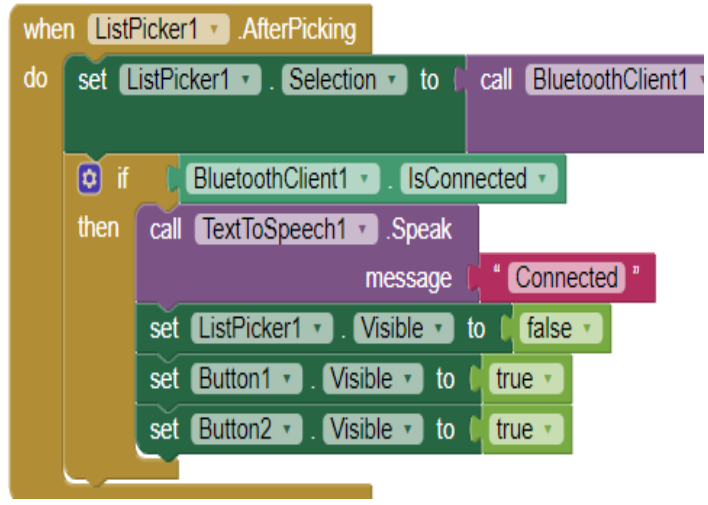

If the connection between the Bluetooth devices cannot be established, the application returns an error (according to the code presented in Fig. 6).

The string that is sent via Bluetooth is formed using "Spinner" lists. These take numerical values between 0 and 12 selected by the user. The values string represents the day of the week and number of hours when the outlet will be active: LUxMAxMIxJOxVIxSAxDUx.

By pressing "Date and time", the system's date and time are configured, by using the device's clock. The display of the date and time is performed using the "join" function, in the form "day of the week ZZ/MM/YYYY hh:mm".

By pressing "SEND PARAMETERS" (Button2), the labels' texts are sent via Bluetooth to the microcontroller and in place of the previously defined texts will appear successful data transmission messages. 
The second software application is implemented on an Arduino development board and offers support for receiving the string sent by the Android application via Bluetooth. The received values become the reference dates for measuring the consumption. In order to prevent any date and time desynchronizations, a backup solution was chosen to provide the correct values for these dates using the real-time clock DS1307. This real-time clock communicates with the Arduino via an I2C channel. The connection or disconnection of the power supply to the monitored consumer is performed with an electromechanical relay that is driven by an $\mathrm{ON} / \mathrm{OFF}$ digital signal. An AC720 current sensor was chosen to monitor the consumption. Using the energy consumption data of the multimedia systems, one can set the current value from which the single-phase outlet will start to be monitored and controlled. The display was integrated in order to validate the programming.

Figure 7. Electrical schematic used to simulate the system

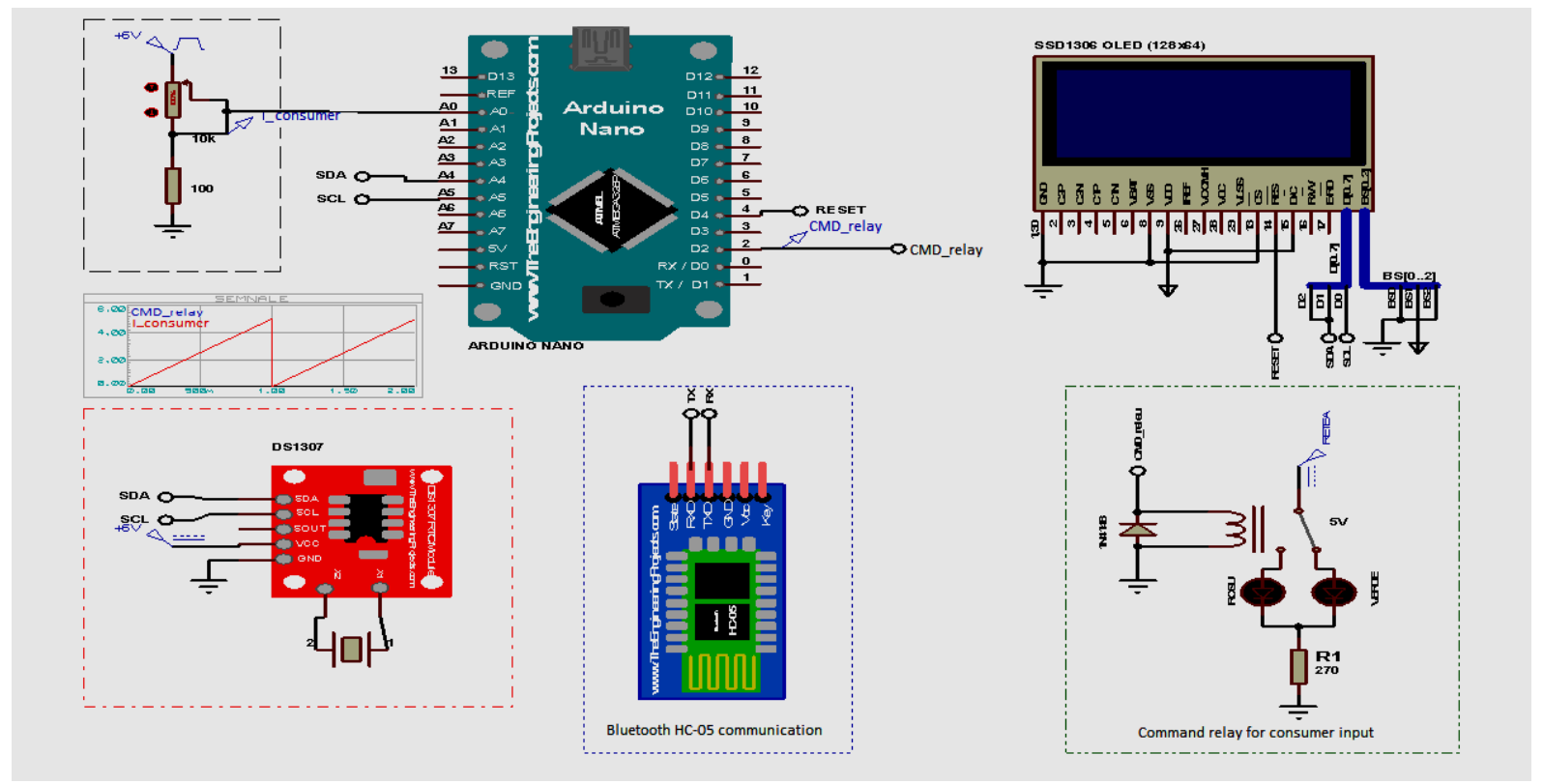

The system was simulated in order to validate the design (see Fig. 7). This simulation was implemented in ISIS Proteus. Labcenter Electronics does not provide libraries for Arduino development boards, but these can be downloaded freely from other web platforms. During the simulations, we have monitored the software application inside the Atmel microcontroller with specific focus on: communication with the graphical LCD, synchronization with the realtime clock, relay command.

Figure 8. Date and time synchronization

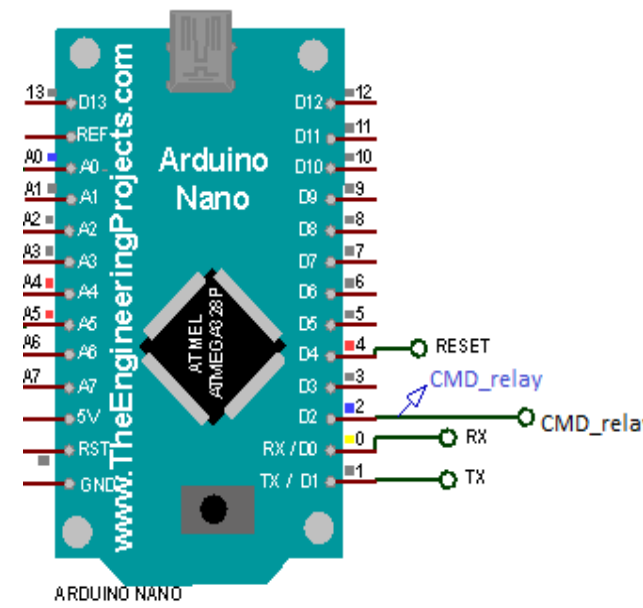

In order to verify the synchronization of the date parameters, the Android application sends a string that configures the operating parameters of the real-time clock. Afterwards, these parameters are displayed on the LCD (see Fig. 8).

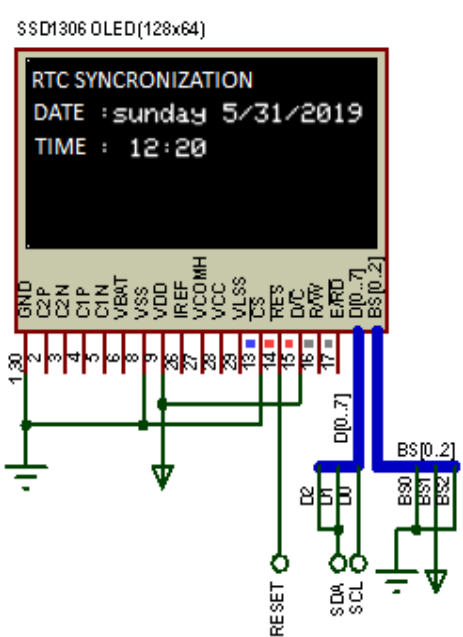

In order to simulate an energy consumption, we have used a ramp variable voltage source in place of the current sensor. The parameters shown in the simulation are not real and only represent the implementation of the requirements. 
Figure 9. Consumer switched on, operation within the time interval, relay switched on

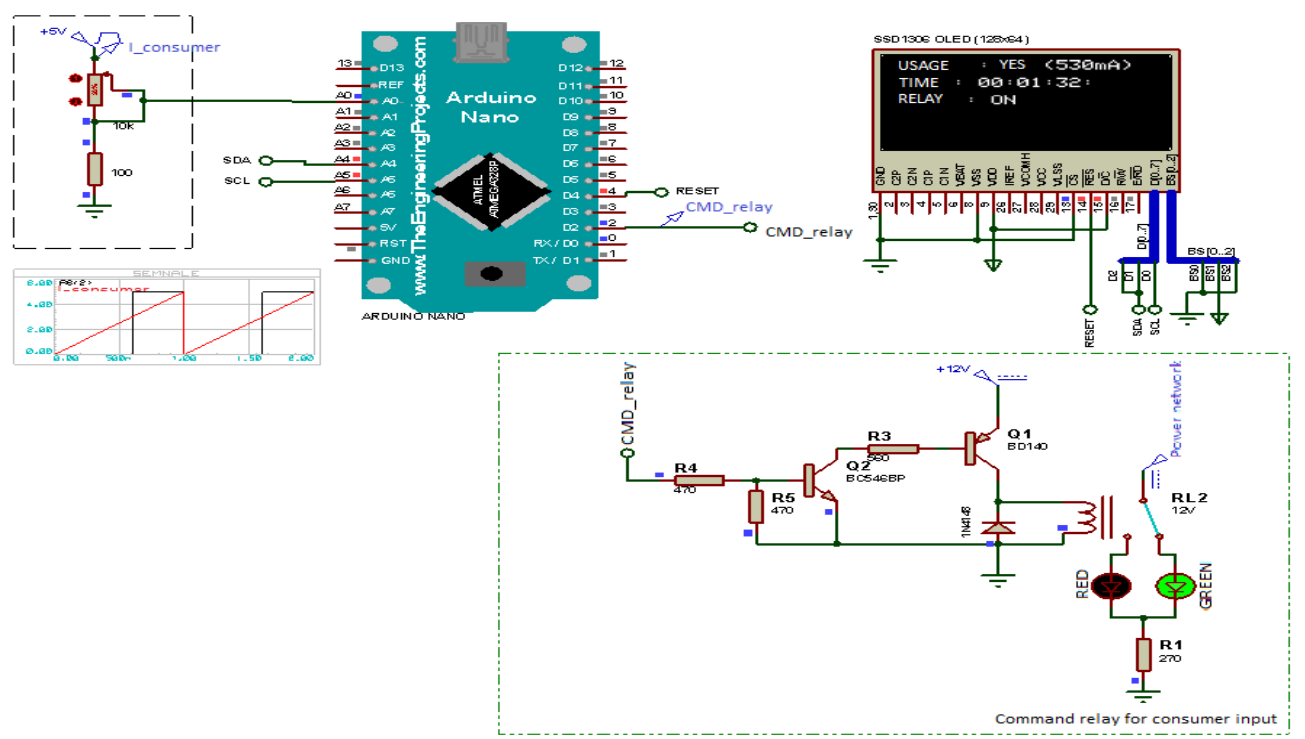

Fig. 9 presents the case when the consumer is switched on and the operating time limit is not passed. Consequently, the relay is switched on.

Figure 10. Consumer switched on, operating time limit passed, relay switched off

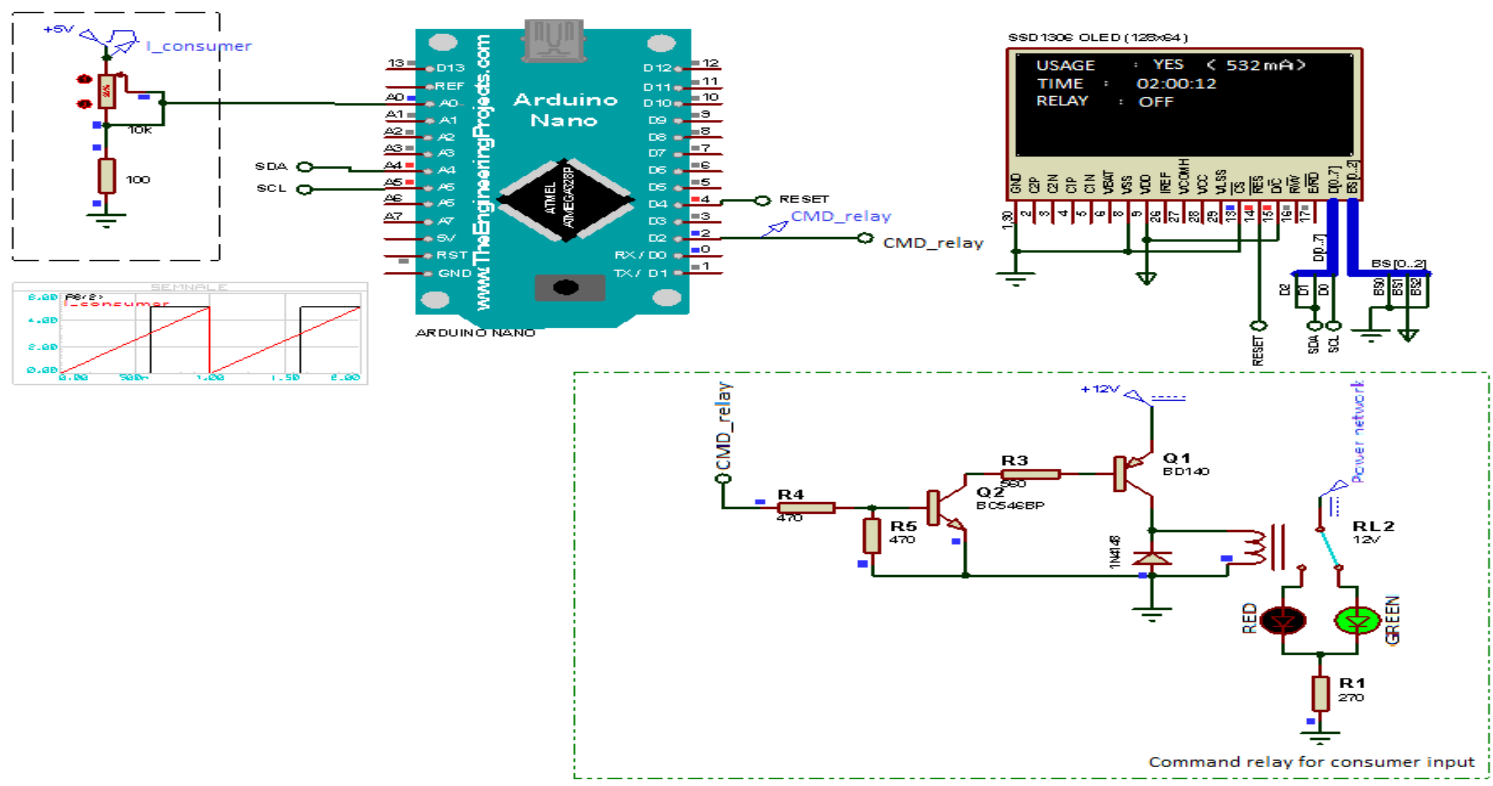

Fig. 10 presents the case when the consumer is switched on, but the operating time limit is passed. Thus, the relay is not switched on. It will be activated starting with the following day.

Figure 11. Consumer switched on, operating time limit passed, relay switched on

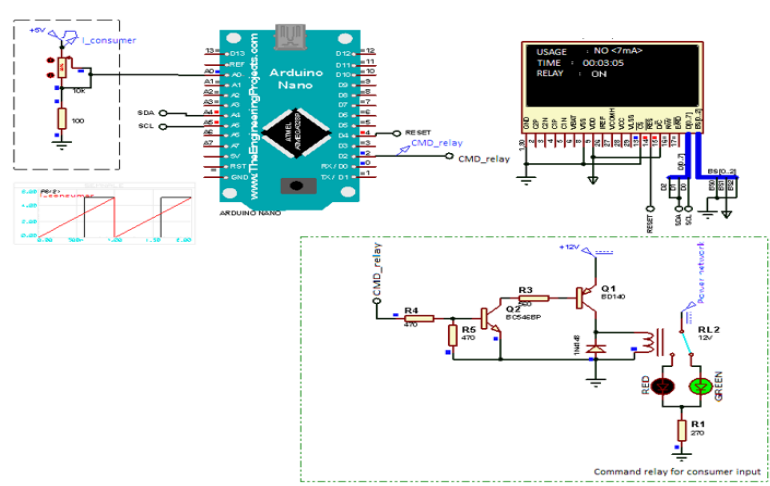


Fig. 11 presents the case when the consumer is switched off and the operating time limit is not passed. Thus, the relay is switched on.

Figure 12. Breadboard prototype of the designed system

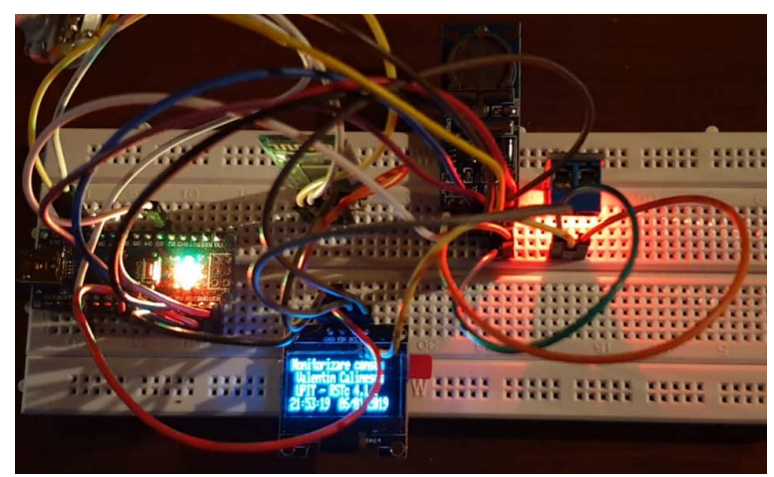

The breadboard prototype (Fig. 12) has answered all the design requirements. In case of industrial manufacturing, the use of SMD components is recommended for limiting the system's size. Also, the LCD display can be removed.

\section{CONCLUSIONS}

Nowadays, the electrical energy consumption increases with the evolution of electronic equipment. Thus, a research direction is focused on finding alternate electrical energy usage optimization solutions. By reducing the time spent in front of multimedia devices, the quality of life is improved by avoiding sedentariness (and, implicitly, obesity) and other health problems. The development of this application was started as a requirement from some parents who wanted to limit their children's access to the TV, game console and computer. The implementation of this solution must take into account all the existing protection rules applied to electrical equipment.

The idea has direct advantages, but also presents some disadvantages, like the possibility to choose a different power outlet. In order to prevent these situations, the project can be extended using the same idea, with the difference that the practical implementation will include a sleeve that integrates a current clamp applied directly on the consumer's power supply cable.

\section{REFERENCES}

[1] Teychenne, Megan; Stephens, Lena D.; Costigan, Sarah A.; et al., The association between sedentary behaviour and indicators of stress: a systematic review, BMC PUBLIC HEALTH Volume: 19 Issue: 1 Article Number: 1357 Published: OCT 232019

[2] Bonfiglio, Natale Salvatore; Renati, Roberta; Pessa, Eliano; The Use of Brain Computer Interface (BCI) Combined with Serious Games for Pathological Dependence Treatment, SYSTEMICS OF INCOMPLETENESS AND QUASISYSTEMS Book Series: Contemporary Systems Thinking Pages: 317-324 Published: 2019

[3] Oliveira, Thyciane Santos; Da Silva Barreto, Lais Karla; ElAouar, Walid Abbas; et al., Computer addiction, internet, social networks? Dependence on the cell phone? The impact of new technologies on individuals' daily lives, RAEREVISTA DE ADMINISTRACAO DE EMPRESAS Volume: 57 Issue: 6 Pages: 634-635 Published: NOVDEC 2017

[4] Obach, I. I.; Abouellail, A. A.; Soldatov, A. I.; et al. Monitoring of Power Supply, International Siberian Conference on Control and Communications (SIBCON), Tomsk State Univ Control Syst \& Radioelectron, Tomsk, RUSSIA Date: APR 18-20, 2019

[5] Li, Zong; Mei, Hongwei; Wang, Liming, A Power Supply Technology for a Low-Power Online Monitoring Sensor Based on Electric Field Induction, SENSORS Volume: 19 Issue: 9 Article Number: 2169 Published: MAY 12019

[6] Xiong Jun-jun; Huang Hua; Deng Xiao-pin; et al. Design of Power Supply State Monitoring System for Active Electronic Current Transformer, China International Conference on Electricity Distribution (CICED) Location: Tianjin Univ, Tianjin, PEOPLES R CHINA Date: SEP 17-19, 2018

[7] https://www.dedeman.ro/ro/priza-programabila-mecanicatimer-01-w-16a/p/1002829

[8] https://www.emag.ro/priza-programabila-digital-ip20-3680w-programare-saptamanala-home-0743h/pd/DG3VX9BBM/

[9] https://www.dlink.com/en/products/dsp-w115-mydlink-wi-fismart-plug

[10] http://www.robofun.ro/arduino.

[11] http://www.atmel.com/Images/doc8025.pdf.

[12] http://ai2.appinventor.mit.edu/\#5351208775712768.

[13] http://appinventor.mit.edu/explore/\#. 